\title{
tic\&société
}

Vol. 13, No 1-2 | 1er semestre 2019 - 2ème semestre 2019 Les industries culturelles à la conquête des plateformes?

\section{Une plateforme au service d'un monde professionnel mobilisé : enjeux symboliques et économiques}

Marie-Eva LESAUNIER

\section{CpenEdition}

Journals

Édition électronique

URL : http://journals.openedition.org/ticetsociete/3299

DOI : 10.4000/ticetsociete.3299

Éditeur

Association ARTIC

Édition imprimée

Pagination : 225-246

\section{Référence électronique}

Marie-Eva LESAUNIER, « Une plateforme au service d'un monde professionnel mobilisé : enjeux symboliques et économiques ", tic\&société [En ligne], Vol. 13, N 1-2। 1er semestre 2019 - 2ème semestre 2019, mis en ligne le 20 avril 2019, consulté le 15 mai 2019. URL : http:// journals.openedition.org/ticetsociete/3299; DOI : 10.4000/ticetsociete.3299 
tic\&société - 13(1-2), 2019

\title{
Une plateforme au service d'un monde professionnel mobilisé : enjeux symboliques et économiques
}

\begin{abstract}
Marie-Eva LESAUNIER
Marie-Eva Lesaunier est doctorante en sciences de l'information et de la communication au Centre d'analyse et de recherche interdisciplinaire sur les médias (CARISM, université Paris 2 Panthéon-Assas). Ses recherches portent sur les conditions de production des documentaires en France dans une perspective socioéconomique. Elle travaille également sur une sociologie du journalisme, de l'insertion aux pratiques professionnelles. marieevalesaunier@gmail.com
\end{abstract}


Une plateforme au service d'un monde professionnel mobilisé : enjeux symboliques et économiques

\section{Une plateforme au service d'un monde professionnel mobilisé : enjeux symboliques et économiques}

Résumé : En prenant pour objet une plateforme de vidéos à la demande par abonnement spécialisée dans le documentaire de création, cet article cherche à rendre compte des enjeux professionnels d'une telle entreprise, enjeux qui recouvrent des dimensions à la fois symboliques, politiques, économiques et industrielles. II apparaît que le modèle économique de la plateforme, fondé sur l'intégration verticale de la filière de production des films, témoigne de la mobilisation d'un monde professionnel pour la défense et la réaffirmation de sa légitimité et de ses frontières. Ce modèle, très éditorialisé, est néanmoins confronté à un écosystème numérique concurrentiel où des logiques préexistantes restent à l'œuvre. La viabilité des plateformes audiovisuelles de niche est donc mise en question.

Mots-clés : documentaire, plateforme, mobilisation professionnelle, niche, production, diffusion.

Abstract: This paper focuses on professional issues associated with the creation of a subscription-based on-demand video platform that specializes in creative documentaries. These issues involve symbolic, politic, economic and industrial dimensions. Based on the vertical integration of the film production industry, the platform's business model both expresses and relies upon the mobilization of a professional social world to protect and assert its legitimacy and borders. However, this highly editorialized model must function within a competitive digital environment where preexisting logics are at work. This raises questions about the viability of niche platforms.

Keywords: documentary, platform, professional mobilization, niche, production, broadcast.

Resumen: Mediante el análisis de una plataforma de videos a la carta por suscripción especializada en documentales de creación, este artículo busca estudiar los retos profesionales de tal empresa; retos que simultáneamente abarcan las 


\section{Marie-Eva LESAUNIER}

dimensiones simbólicas, políticas, económicas e industriales. De hecho, el modelo económico de la plataforma, fundado en la integración vertical de la producción de películas, muestra la movilización defensiva del mundo profesional y la reafirmación de su legitimidad y de sus fronteras. Este modelo, muy editorializado, se encuentra, sin embargo, confrontado a un ecosistema digital competitivo, en donde las lógicas preexistentes continúan existiendo. Entonces, la viabilidad de las plataformas audiovisuales de nicho es puesta en cuestión.

Palabras claves: documental, plataforma, movilización profesional, nicho, producción, difusión. 
Une plateforme au service d'un monde professionnel mobilisé : enjeux symboliques et économiques

\section{Introduction}

Le contexte politique et économique de la filière documentaire en France en 2014-2015 incite un certain nombre de professionnels à lancer le projet d'une plateforme numérique de vidéo à la demande spécialisée dans le documentaire de création: Tënk. La fin de l'année 2014 est marquée par l'annonce du Centre national du cinéma et de l'image animée (CNC) d'une réforme du Compte de soutien aux industries de programmes (Cosip) et d'un contrôle accru exercé sur les films financés avec l'apport des chaînes de télévision locales. L'apport minimum d'une chaîne de télévision pour déclencher les aides publiques automatiques à la production des œuvres s'élevait (et s'élève encore) à 12000 euros en numéraire par heure de programme. Peu de télévisions locales apportaient effectivement cette somme, déguisant des apports en industrie (montage, mixage, étalonnage) en apport numéraire et générant ainsi des budgets faussés, ce qui permettait à des films dits «fragiles » (CNC, 2018b) d'obtenir un soutien automatique.

Cette initiative du CNC remettait ainsi en cause le fonctionnement - bricolé - d'une filière industrielle, d'une niche audiovisuelle, celle du documentaire de création. L'annonce de cette réforme a contribué à déclencher une mobilisation professionnelle prenant d'abord la forme d'un collectif nommé "Nous sommes le documentaire ». Une tribune éponyme est publiée dans Le Monde en mars 2015 et un long cycle de négociation s'ouvre avec le CNC et les partenaires professionnels. C'est dans ce contexte que naît le projet d'une plateforme spécialisée. Son instigateur principal expliquait, lors d'un entretien en 2016 :

"C'est vrai qu'il y a trois ans, jamais, jamais je n'aurais imaginé travailler, moi, avec des copains, à monter une plateforme et une télé, quoi, jamais! Ça me paraissait... Parce que dans les années 1980 justement, on s'est posé la question, on s'est dit, est-ce que nous, on doit monter une télé, nous les producteurs indépendants ? On se dit non, puisqu'en face, on a des partenaires télé qui jouent le jeu [...]. Donc je n'imaginais pas qu'il y ait besoin d'une 
plateforme. Et puis il y a eu un faisceau d'événements, de situations, qui justement nous ont fait basculer, en nous disant, mais, il faut prendre notre destin en main, parce que là, c'est foutu quoi, foutu de foutu. " (Auteurréalisateur, producteur, fondateur de Tënk, programmateur et cofondateur des États généraux du film documentaire)

En août 2016 est officiellement lancée la plateforme de vidéo par abonnement dédiée au documentaire d'auteur Tënk. Cette plateforme de niche est en filiation avec les Rencontres Tënk fondées en 2002, qui consistent à créer un réseau mondial de documentaristes en rassemblant " des producteurs des pays d'où proviennent les auteurs ainsi que des producteurs européens et des diffuseurs TV devant lesquels les auteurs sélectionnés avec leur producteur national présentent leurs projets ${ }^{1}$ ». Au contraire d'une simple mise à disposition du contenu, la plateforme se veut très éditorialisée et affirme son positionnement du côté du " documentaire d'auteur » et de sa défense, autant dans les contenus sélectionnés par les professionnels chargés des "plages ", que dans le discours d'escorte qui l'accompagne.

Cette mobilisation et la création de Tënk rendent visible un monde professionnel au sens de Strauss (1992), ses frontières et les négociations autour de celles-ci. Ainsi, l'originalité de cet article réside dans le fait qu'il n'envisage pas le rapport entre plateformes et industries culturelles dans une dimension conflictuelle ou compétitive. Au contraire, la plateforme apparaît ici, pour les professionnels, comme un instrument d'émancipation, la réponse d'un monde professionnel à une déviance subie (Becker, 1985).

Le documentaire de création est caractérisé par l'affirmation d'un regard d'auteur, d'une subjectivité venant se saisir d'un aspect de la réalité. II s'oppose en ce sens au reportage journalistique qui tend vers un idéal d'objectivité (BarreauBrouste, 2013). La façon dont les professionnels du documentaire de création définissent leur pratique repose en partie sur des oppositions construites entre objectivité et subjectivité, reportage et film, journaliste et auteur. Ces

1 Site Internet de DocMonde, réseau international de formation au documentaire : http://www.docmonde.org/ (consulté le 22 août 2018). 
Une plateforme au service d'un monde professionnel mobilisé : enjeux symboliques et économiques

tensions, incarnées notamment dans la lutte contre le "formatage " des documentaires télévisés, forment une rhétorique qui structure le monde professionnel depuis une quarantaine d'années (Barreau-Brouste, 2011, 2013 ; Kilborne, 2011 ; Sauguet, 2007). Émilie Sauguet a mis en évidence la période des années 1980-1990, au cours de laquelle les professionnels du documentaire se sont organisés en vue de légitimer leurs pratiques et leurs œuvres, en refondant une définition du documentaire. Elle fait notamment référence aux créations de La Bande à Lumière en 1985, des États généraux du documentaire de Lussas en 1989 et de l'association Addoc en 1992, qui participent du processus de définition d'un genre avec l'élaboration de la notion de document ire de cré tion. Cette période consiste en effet en la construction d'une identité professionnelle « autour de l'idée que le documentaire constitue un genre cinématographique à part entière, auquel le circuit de la télévision ne donne pas la place qu'il mérite " (Sauguet, 2007, p. 34). La mobilisation professionnelle de 2015 s'inscrit donc dans ce processus avec non plus un enjeu définitionnel, mais économique et politique.

Notre article est fondé sur une approche socioéconomique. En ce sens, nous adoptons une perspective interactionniste nous amenant à envisager le documentaire de création comme un monde social et, plus précisément, comme un micro-monde professionnel (Strauss, 1992). Si d'un point de vue structurel, la mise en cause des conventions et de la hiérarchie qu'elles impliquent inscrit les professionnels dans le pôle dominé du champ documentaire (Bourdieu, 1992), l'approche par le monde social semble féconde, car elle invite à s'intéresser davantage aux mouvements au sein de et entre des mondes professionnels de diverses natures et de diverses tailles. Ce faisant, Tënk apparaît comme la matérialisation concrète et symbolique d'un mouvement s'opérant au sein du micromonde. Allant dans le sens d'une redéfinition des frontières, la mise en œuvre de la plateforme traduit un mouvement du monde de l'art qui tend vers une "révolution " (Becker, 1982, p. 308), entendue comme un changement « de nature à modifier les réseaux de coopération » (p. 305). La mobilisation et la création de la plateforme incarnent ainsi cette

double offensive contre le fonctionnement normal du monde de l'art concerné. Au plan des idées, elle prend la 
forme de manifestes, de textes critiques, de remises en cause esthétiques et philosophiques [...]. Au plan de l'organisation, elle vise à la mainmise sur les sources de financement, les publics et les systèmes de distribution (Becker, 1982, p. 305).

À ce socle théorique, nous maillons une approche économique portant sur l'analyse du rapport entre industries culturelles et numérique (Benghozi, 2003 ; Benghozi et Benhamou, 2008; Benghozi et Paris, 2006; Mairesse et Rochelandet, 2015). En interrogeant notamment les concepts de longue tr’ îne (Anderson, 2007), d'oligopole à fr'nge (Reynaud-Cressent, 1982) et leurs limites, nous nous efforçons de complexifier et de nuancer l'analyse de l'objet ainsi construit. Cette double approche, sociologique et économique, réaffirme la richesse analytique de l'interdisciplinarité propre aux sciences de l'information et de la communication.

Le présent article tente donc de saisir dans quelle mesure la plateforme Tënk est le reflet d'un changement au sein d'un pan des industries culturelles, envisagé comme un monde professionnel en quête de réaffirmation, voire de reconstitution de ses frontières.

En s'intéressant à une niche de l'industrie de l'audiovisuel, l'analyse du rapport entre celle-ci et les plateformes numériques rend compte d'enjeux sociologiques et économiques encore peu mis en évidence du fait de l'intérêt porté aux modèles dominants. La plateforme devient, pour les acteurs étudiés, un instrument d'émancipation à forte dimension symbolique.

Nos résultats sont construits à partir de l'analyse de dix-huit entretiens semi-directifs menés avec des professionnels du documentaire de création (producteurs et/ou réalisateurs, diffuseurs). Ils sont complétés par l'analyse discursive de la communication de la plateforme (site internet, diffusion de courriels), ainsi que par l'observation de plusieurs réunions de présentation de Tënk ayant eu lieu lors des États généraux du film documentaire de Lussas en août 2016, 2017 et 2018 ainsi qu'au Forum des images à Paris en septembre 2016.

La première partie des résultats met en évidence la forte dimension symbolique associée à la plateforme Tënk. Celle-ci est alors envisagée et revendiquée comme un projet culturel et 
Une plateforme au service d'un monde professionnel mobilisé : enjeux symboliques et économiques

politique au secours d'une filière fragilisée par les logiques industrielles à l'œuvre dans l'audiovisuel. La seconde partie interroge plus spécifiquement la dimension économique du projet à travers laquelle sont saisies les conditions de l'émancipation initiée par le monde professionnel.

\section{La plateforme " au secours » d'un monde professionnel mobilisé ?}

La création de Tënk revêt une forte dimension symbolique de réaffirmation de l'existence du micro-monde du documentaire de création, de ses spécificités et de sa légitimité.

\subsection{Un continuum avec une mobilisation professionnelle}

Le contrôle des financements des chaînes de télévision locales s'apparente à une phase d'étiquetage d'une pratique professionnelle du documentaire jugée déviante dans ses modes de production (Becker, 1985). II apparaît un sentiment d'exclusion, de marginalisation parmi les professionnels interrogés. La déviance, comme l'explicite Becker (1985) est le résultat d'une interaction et s'incarne à la fois dans l'étiquetage d'une action comme transgressive et dans l'intériorisation de cette action comme transgressive chez ledit déviant. Cette " déviance subie » transparaît dans les entretiens au regard de deux dimensions. D'une part, elle se manifeste dans le rapport aux chaînes de télévision nationales, clé de voûte du financement des films, mais dont le désengagement vis-à-vis du documentaire est vivement critiqué :

«On est prisonnier de la demande, et on n'arrive plus à faire nos films. " (Producteur)

"Sur des films qui ne sont pas prêts à faire trop de concessions sur la forme, ni sur le fond, le dialogue ne passe pas. On ne communique pas. " (Producteur)

"II y a une injustice profonde à vouloir stigmatiser l'ensemble des télédiffuseurs et l'ensemble des producteurs indépendants qui travaillent avec ces chaînes de télévision locales. » (Producteur) 
« Si tu as assisté à ce débat au Réel, je ne sais plus exactement les termes de [représentant du $\mathrm{CNC}$ ], mais on était quasiment des escrocs, qui utilisions un système d'optimisation. " (Producteur)

D'autre part, la déviance s'incarne également par rapport aux décisions prises par le CNC quant à la réforme du Cosip. En effet, tout en prenant en charge les spécificités du documentaire de création, les mécanismes de soutien semblent en même temps le cantonner dans une économie fragile. Puisque la coopération routinière se fait avec les télévisions locales de par un investissement moindre des télévisions nationales dans le documentaire de création, et que ces chaînes locales ne peuvent pas financer une œuvre à hauteur du seuil minimum requis pour obtenir l'aide automatique du Cosip, ces œuvres sont, depuis la réforme de 2015, cantonnées à l'aide sélective à la production.

Les diffuseurs, quelle que soit leur nature, représentent près de la moitié $(49,1 \%)$ des financements des documentaires audiovisuels (CNC, 2018a). En 2016, l'apport des chaînes nationales publiques s'élève à 141 millions d'euros, contre 3 millions pour les chaînes de télévision locales (CNC, 2017b). Cet écart laisse entrevoir la forte disparité des modes de production au sein du monde du documentaire. Si la part des aides du CNC s'élève à $19,6 \%$ du financement des films en 2017 (CNC, 2018a), elle peut parfois être portée à $80 \%$ du budget total d'un film (en prenant en compte les autres financements publics provenant des fonds régionaux). D'ailleurs, ce seuil dérogatoire d'intensité d'aides publiques est réservé aux documentaires de création et ne permet d'accéder qu'à un soutien sélectif (en automatique, la dérogation peut être portée de $50 \%$ à $60 \%$ d'aides publiques). Ces documentaires sont alors qualifiés "d'œuvres fragiles et à petit budget ", c'està-dire inférieurs à 150000 euros par heure produite (CNC, $2018 b)$. Enfin, le montant du soutien généré dans le cadre de l'aide automatique est fonction de l'apport horaire d'un diffuseur : plus celui-ci est élevé, plus le soutien le sera aussi. Le micro-monde et les œuvres qui y sont réalisées se voient dès lors généralement privés d'une certaine stabilité permise par l'accès à un compte de soutien automatique, intrinsèquement structurant, puisqu'il garantit aux producteurs une possibilité d'investissement dans des œuvres ultérieures : 
Une plateforme au service d'un monde professionnel mobilisé : enjeux symboliques et économiques

" On a une sorte de reconnaissance symbolique très forte. Mais par contre, on n'existe pas du tout dans l'économie. » (Producteur)

«Et le problème des petits apports des chaînes locales, c'est que les soutiens qu'on va demander, on ne peut pas demander beaucoup non plus. Donc ça limite un peu, ça fait des films à petits budgets. " (Producteur)

« Je pense qu'on remplit des missions de service public. Les financements ne sont pas à la hauteur de ce travail-là, quoi. " (Producteur)

Le conflit et la mobilisation autour de la réforme du Compte de soutien en 2015 sont donc révélateurs de cette « déviance subie ". C'est dans ce contexte que le projet de la plateforme de vidéos à la demande par abonnement prend forme. Constatant la « rupture » avec l'industrie audiovisuelle incarnée par les chaînes nationales, des professionnels cherchent à recréer des conventions officielles qui leur sont favorables (Becker, 1982). Un des fondateurs de la plateforme se positionne dans ce paradigme de la rupture entre documentaire de création et chaînes nationales de télévision. Le verb` tim cidessous explicite le continuum entre réforme, mobilisation et initiation du projet de plateforme :

"Les films coproduits par les télés locales, c'est à peu près 300 films par an. Et en gros sur ces 300 films, c'est quand même dans l'ensemble du documentaire assez créatif, et il y a 150 films, la moitié, qui sont vraiment de très bon niveau. lls ne peuvent pas se faire ailleurs, les gens mettent beaucoup d'énergie, il n'y a pas beaucoup de moyens, mais voilà. Et c'est surtout le moyen de faire advenir de jeunes auteurs, de permettre à des jeunes producteurs de faire leurs armes, de rentrer dans le métier, d'apprendre [...]. Quand on a démarré en 1989 les États généraux, il $y$ avait 100,150 producteurs ; aujourd'hui, il y en a 700-750. Mais normalement il n'y aurait pas dû $y$ avoir de problème, puisqu'il $y$ avait floraison de télés qui sont nées depuis. Sauf que ces télés ne veulent plus du documentaire d'auteur. Donc du coup, il y a beau y avoir un nombre énorme, il n'y a plus de place pour le documentaire d'auteur [...]. On s'est dit qu'il y a une opportunité historique, c'est le Net. Pourquoi on 


\section{Marie-Eva LESAUNIER}

laisse Mubi, pourquoi on laisse Netflix, pourquoi on laisserait ça uniquement à des entrepreneurs de l'audiovisuel, on est capable de faire ça, les auteurs, on en connaît beaucoup, les festivals, c'est tout un réseau, on les connaît, on est une centaine dans le réseau de Lussas à avoir même des regards très pointus sur des champs du film, la danse, par exemple, l'architecture, la musique, tout ça, c'est des richesses qui dorment, donc allons-y. Tentons. Construisons un bâtiment, montons un projet. Donc on est parti sur un projet, à Lussas et on se dit, on n'a pas d'argent, mais devenons des industriels au même titre que les autres, avec une volonté, c'est qu'on soit indépendant et que cet outil soit aux mains des indépendants et défende la création. Voilà, indépendance et création. Et monde. » (Auteur-réalisateur, producteur, fondateur de Tënk, programmateur et cofondateur des États généraux du film documentaire)

La plateforme apparaît ainsi comme un instrument d'émancipation, de défense d'un micro-monde professionnel dans un contexte de difficultés économiques et de remises en causes symboliques.

\subsection{Un projet culturel et politique contre « l'industrie culturelle »}

La forte dimension symbolique de la plateforme spécialisée est particulièrement observable si on analyse les discours produits par les enquêtés lors des entretiens et dans la communication de Tënk. Ceux-ci mettent en évidence, d'une part, un discours sur le rôle des films que les professionnels réalisent, produisent et diffusent et, d'autre part, sur l'industrialisation de l'audiovisuel et ses conséquences. En ce sens, et d'un point de vue compréhensif, ces discours se rapportent à la pensée critique de l'École de Francfort (Adorno et Horkheimer, 1947 ; Voirol, 2011). " II y a une dérive vers une marchandisation de plus en plus forte de l'image ", estime un producteur.

La conception des professionnels des œuvres qu'ils produisent est caractérisée par l'affirmation de leur importance au sein de la société, permettant aux publics d'exercer leur regard et leur intelligence. Au contraire, les documentaires 
Une plateforme au service d'un monde professionnel mobilisé : enjeux symboliques et économiques

diffusés en télévision sont décrits, de façon générale, comme ne remplissant que partiellement cette fonction, associée à la mission de service public :

" Je pense qu'il faut absolument se donner les moyens pour que ce secteur-là perdure, parce que je pense que c'est pour le coup essentiel pour avoir le recul nécessaire et la réflexion nécessaire sur tout ce qui nous arrive, avec d'autres champs hein, le spectacle vivant, la littérature, la recherche [...]. » (Réalisateur)

"Nos désirs de films viennent de nous, notre rapport au monde, avec la croyance qu'il y a quelque chose qui est hyper important à dire. » (Réalisateur)

«Tu as toujours l'impression de voir la même chose, de voir des trucs qui sont sans danger, dans tout ce que tu regardes, tu sens qu'on ne prend pas de risque et puis après, politiquement, les films ne disent rien, c'est très fade tout ça. " (Productrice)

Ces définitions font écho aux penseurs de l'approche critique (Adorno, 1971; Benjamin, 1936), qui considéraient le rapport à une œuvre culturelle comme une expérience esthétique contenant une portée politique dans le processus d'appropriation de l'œuvre par ses spectateurs.

"Loin d'éclairer et d'exprimer l'expérience mutilée des sujets et les rapports sociaux dans lesquels ces derniers sont inscrits, elle [la culture industrialisée] fait mine de les considérer, sinon de les dénoncer, sans rien ajouter à leur intelligibilité ; au contraire, elle les rend plus opaques. Bien au contraire, au lieu de permettre une transfiguration esthétique de la souffrance humaine appelant un autre rapport au monde, elle incite à l'acceptation du malheur sous couvert de plaisir - facile d'accès - d'où son caractère mythologique. » (Voirol, 2001, p. 143.)

On le voit, les professionnels du micro-monde du documentaire de création partagent cette conception politique forte des films qu'ils produisent et réalisent. Si cette référence théorique n'est pas explicitement revendiquée, contrairement à l'analyse proposée par Sophie Noël (2012) à propos du secteur de l'édition indépendante, elle n'en est pas moins remarquable et systématique, tant chez les enquêtés que dans l'ethos 
construit par le discours de la plateforme sur elle-même. L'extrait de l'éditorial mis en ligne sur le site internet témoigne de cet ancrage critique affirmé :

L'architecture de Tënk est accidentée; complexe et polymorphe elle ressemble à son époque, mais s'en différencie par son refus de croire à une norme et à l'idée d'un grand public qu'elle pourrait contenir partageant les mêmes attentes. II y a autant de spectateurs que de façon de recevoir ou de créer un film. Les plages de Tënk sont des espaces de rencontres, au croisement de regards et de façons de penser le monde ${ }^{2}$.

La mise en discours de la plateforme réaffirme ainsi l'aire culturelle (Shibutani, 1955) partagée par le monde professionnel, sa légitimité et ses frontières. En ce sens, Tënk devient en quelque sorte un nouveau lieu symbolique (lieu symbole) (Ripoll, 2005). Ce projet s'inscrit bien dans une logique d'émancipation, de mobilisation d'acteurs se saisissant du numérique et de l'écosystème des plateformes pour réaffirmer leur existence et leur légitimité, et ce, tant symboliquement, comme nous venons de le voir, qu'économiquement.

\section{Le changement du monde professionnel en question}

L'économie numérique désigne les transformations des activités économiques et sociales portées par la diffusion massive d'une technologie "générique », le numérique, qui engendre un ensemble d'opportunités nouvelles dont peuvent s'emparer avec plus ou moins de succès les individus et les organisations. (Mairesse et Rochelandet, 2015, p. 225.)

La mise en place de la plateforme s'appuie sur un modèle économique original 'priori à même de favoriser la diffusion et la production des documentaires de création. II apparaît néanmoins que la niche audiovisuelle qu'est ce genre présente quelques limites quant à la viabilité d'un tel modèle.

\footnotetext{
2 Tënk, Édito, 2018 : www.tenk.fr (consulté le 20 juillet 2018).
} 
Une plateforme au service d'un monde professionnel mobilisé : enjeux symboliques et économiques

\section{1 «Le modèle économique de Tënk repose sur l'engagement de ses abonnés ${ }^{3}$ "}

Tënk compte aujourd'hui 6500 abonnés, parmi lesquels $85 \%$ sont « très familiers du documentaire, et $40 \%$ travaillent dans ce milieu ${ }^{4}$. Le lien entre le monde professionnel et l'abonnement est donc ténu. De plus, il apparaît que l'investissement financier dans le projet constitue une forme d'engagement pour le documentaire. L'acte d'abonnement à la plateforme est en effet explicitement revendiqué comme tel. La construction discursive de la communication de Tënk semble s'attacher à lier abonnement et engagement au service du genre, comme en atteste l'extrait suivant, issu d'un courriel envoyé par l'association Ardèche Images en juillet 2017 :

"Nous savons que le temps peut manquer parfois: "Pourquoi m'abonner si je ne regarde pas de films ? J'ai déjà tant de livres/journaux/travail en attente !". Aujourd'hui, nous voulons vous convaincre que s'abonner à Tënk est aussi un acte militant : c'est s'engager à nos côtés pour donner un avenir au documentaire d'auteur, celui qui vous interpelle, qui vous fait rêver, qui vous dérange et qui manque tant dans le paysage audiovisuel d'aujourd'hui... » (Ardèche Images, "L'avenir de Tënk ne se fera pas sans vous ! », 11 juillet 2017.)

L'abonnement n'est ainsi pas envisagé comme un simple acte de consommation où offre et demande se rencontrent, mais comme une participation concrète aux redéfinitions des frontières mises en œuvre par la plateforme. Plus encore, les abonnés se voient attribuer un rôle d'ambassadeurs et représentent une part importante de la stratégie de communication de Tënk (dont le budget est limité), fondée notamment sur le bouche-à-oreille. Matérialisant cette dynamique de prescription, la possibilité est donnée aux abonnés d'offrir des abonnements ou un film à des nonabonnés. La plateforme qualifie ainsi ses abonnés de " contributeur-rice ", de " ambassadeur-rice », de " réseauteur-rice », de " messager-ère », de " donateur-rice »,

\footnotetext{
3 «Pour la suite de Tënk », éditorial consulté le 10 juin 2018 : https://www.tenk.fr/p/objectif10000.

4 Tënk, Enquête interne par questionnaire réalisée auprès des abonnés, 2017, données recueillies lors d'une réunion d'étape en août 2018 aux États généraux du film documentaire.
} 
de «juré-e », en fonction des actions de communication encouragées ${ }^{5}$.

Par ailleurs, la première collecte de fonds de Tënk s'est notamment appuyée sur une campagne de financement participatif, largement relayée sur les réseaux professionnels (presse spécialisée, associations de professionnels, syndicats, réseaux sociaux). Sa constitution en Société coopérative d'intérêts collectifs (SCIC) corrobore également le fondement politico-symbolique d'un projet par ailleurs industriel et économique. En 2017, la société rouvre son capital et compte ainsi 97 associés, ce qui lui permet de détenir un fonds de l'ordre de 140000 euros, constituant un "second souffle qui a permis de continuer " (Tënk, deux ans après, Réunion publique, États généraux du film documentaire, 20 août 2018).

La mobilisation du monde professionnel vers son émancipation est ainsi objectivée, dans les actes - d'achat -, en amont et en aval de l'avènement de la plateforme.

\subsection{Un modèle économique fondé sur l'intégration verticale de la filière}

Si ce projet est d'abord culturel et, nous l'avons dit, politique, il est également industriel et économique. II constitue une réponse industrielle à des difficultés économiques revêtant des enjeux symboliques forts pour les professionnels. Du point de vue organisationnel, le modèle économique est en effet pensé selon un développement double. Dans un premier temps, il s'agit d'assurer la diffusion de films correspondant à la ligne éditoriale, moyennant un achat de droits auprès de leur détenteur. Ce premier volet a pour ambition de mettre à disposition du public des films dits "invisibles » (Bourreau, Maillard et Moreau, 2015), tout en garantissant une rémunération à leurs ayants droit (le tarif pour l'achat de droits est de l'ordre de 200 à 300 euros par heure). C'est le premier enjeu de la plateforme. Dans un second temps du développement, l'ambition est de participer au financement de la production des films. L'objectif est donc d'être efficient sur l'ensemble de la filière de fabrication des documentaires. En effet, Tënk est dès le départ pensée pour être constituée en service de télévision, ayant des obligations de diffusion et de

${ }^{5}$ Tënk, Soutenir, 2018 : www.tenk.fr (consulté le 20 juillet 2018). 
Une plateforme au service d'un monde professionnel mobilisé : enjeux symboliques et économiques

coproduction d'œuvres originales, devenant un «nouvel acteur de la production de documentaires dans un contexte où les diffuseurs coproducteurs se raréfient " (Tënk, le documentaire d'auteur sur abonnement, dossier de presse, 2018), comme spécifié plus haut.

En inscrivant dans le modèle d'affaires l'investissement dans la production d'œuvres (fabrication) grâce aux revenus générés par les abonnements (diffusion), la fonction d'intermédiation de la plateforme est ici dépassée et l'amont et l'aval de la filière sont envisagés ensemble dans ce qui s'apparente à un « cercle vertueux ». L'intégration verticale de la filière par les professionnels concernés constitue ainsi une forme concrète de mouvement au sein d'un monde professionnel vers un ajustement de ses frontières, dont l'originalité sur le plan économique doit être relevée (Bullich et Guignard, 2014). Comme l'expliquent également Mairesse et Rochelandet, "[l] es recettes tirées des microventes [des grandes plateformes Amazon, Netflix, Spotify] ne garantissent ' priori en rien le financement de nouvelles œuvres indépendantes ou difficiles d'accès et ne permettent pas non plus d'assurer la survie de petites structures de production » (p. 230).

Au contraire, le modèle de Tënk s'inscrit dans une logique où il serait possible de

concilier les fonctions d'appariement et de financement de la culture en générant un nouvel écosystème de diversité créative dans lequel les plateformes accompagneraient les projets artistiques une fois produits; elles pérenniseraient ainsi un ensemble de "réseaux artistiques locaux " [mondes professionnels] reliés selon une logique de "petit monde " qui pourraient coexister avec, voire remettre en cause, les acteurs et modèles économiques dominants (Mairesse et Rochelandet, 2015, p. 230).

\subsection{Une plateforme par et pour un micro-monde social : limites d'une offre numérique de niche}

L'hypothèse avancée ci-dessus par les auteurs est formulée au conditionnel. En effet, si ce modèle est original et semble à même d'exploiter les potentialités offertes par l'économie numérique pour défendre et valoriser la création 
- documentaire -, il est en revanche certain que ces potentialités se trouvent limitées ou contraintes par des logiques à la fois endogènes et exogènes au numérique.

Un tel modèle suppose en effet d'atteindre un seuil d'abonnés suffisant pour financer la production d'œuvres nouvelles. La plateforme Tënk a, dès sa création, fixé son seuil de rentabilité (rendant par-là possible l'investissement dans la coproduction d'œuvres) à 10000 abonnés. La société a réévalué ce taux de rentabilité en 2018, le portant à 8500 abonnés. Ici, la pertinence du modèle éditorial et économique est à interroger. L'offre de la plateforme est une offre de niche, et comme nous l'avons déjà précisé, $85 \%$ des abonnés actuels sont familiers du documentaire. Si le phénomène de longue traîne théorisé par Anderson (2007) supposait une augmentation de la part des produits de niche dans la consommation culturelle globale grâce au numérique, ce modèle a depuis été discuté et nuancé. Ici, la limite est double.

D'une part, il a été démontré que les produits de niche ne bénéficient pas nécessairement de cet effet de longue traîne. Plusieurs limites ont en effet été mises en exergue, notamment la ventilation de la consommation des individus étant effectivement sensibles aux produits de niche entre lesdits produits et les best-sellers (Bourreau, Maillard et Moreau, 2015). La théorie fondée par Anderson reste donc à démontrer dans la mise en œuvre concrète des écosystèmes des plateformes numériques, au sein desquels la domination de "l'effet best-sellers » semble perdurer (Mairesse et Rochelandet, 2015, p. 228). L'un des enjeux majeurs pour la plateforme est donc lié à la problématique des usages. Quelle propension à payer ? Le multi-abonnement est-il réel ? Quels arbitrages en matière de coûts financier et temporel sont réalisés? (Blondiaux et Barneaud, 2015.) En ces termes, la très forte éditorialisation sur laquelle est fondée Tënk est-elle viable?

D'autre part et dans ce cas spécifique, il ne s'agit pas d'une agrégation de produits de niche divers, mais d'une plateforme constituée sur une seule niche culturelle: le documentaire d'auteur. S'il y a bien une augmentation de l'offre de produits de " micro-niche » avec le développement du numérique (Benghozi et Benhamou, 2008), la mise en œuvre de Tënk par 
Une plateforme au service d'un monde professionnel mobilisé : enjeux symboliques et économiques

un monde professionnel et ses caractéristiques de niche ne contiennent-elles pas en germe les limites à un succès effectif et, surtout, à une viabilité économique ? Autrement dit, si le concept de longue traîne est un outil pertinent pour observer le rapport de force entre tête et queue de la traîne de façon globale, il ne semble pas opérant dans une perspective micro ou méso, lorsque l'on considère une niche spécifique et son modèle économique propre.

Puisque ce sont les modèles d'affaires dits " généralistes » qui dominent le marché (Netflix, Amazon) (CNC, 2017 a ; CNC et CSA, 2018), le choix éditorial d'un degré élevé de spécialisation apparaît difficile à tenir. La concurrence entre industries culturelles et plateformes est dès lors dépassée par une concurrence entre plateformes, suivant le modèle de l'oligopole à frange concurrentielle au sein duquel un nombre restreint de groupes aux moyens de production concentrés et importants coexistent avec une multitude de petits groupes plus fragiles (Mairesse et Rochelandet, 2015).

Ces tendances remettent en cause la stratégie de la plateforme et vont dans le sens d'un regroupement de diverses niches dans l'écosystème numérique. C'est d'ailleurs vers des formes de partenariats que s'oriente désormais Tënk. À l'état de projet, il s'agirait de créer une alliance (Strauss, 1992) :

« se regrouper en tant qu'indépendants et faire des propositions globales [...]. On essaye de voir comment on pourrait faire une plateforme que chacun alimenterait, pour construire une quintessence de l'approche d'auteur. On aurait une force de visibilité supplémentaire. " (Auteurréalisateur, producteur, fondateur de Tënk, programmateur et cofondateur des États généraux du film documentaire, réunion d'information Tënk, Lussas, août 2018)

Cette déclaration, faite en août 2018 lors des États généraux du documentaire de Lussas, témoigne de la réaction de la société face aux enjeux concurrentiels et économiques que nous venons d'expliciter. Cette stratégie d'alliance, théorisée par Strauss, est également prégnante lors des mobilisations professionnelles telles que celle évoquée au début de cet article. L'enjeu est alors de dépasser les clivages pour 
mutualiser les problématiques et les revendications ${ }^{6}$. C'est d'ailleurs autour de cette question que certains professionnels doutent de la viabilité du modèle initialement pensé et aujourd'hui en place, rappelant au chercheur, s'il l'avait oublié, qu'un monde professionnel n'est pas homogène, mais traversé par des tensions, des divergences de points de vue, de tactiques et de stratégies.

\section{Conclusion}

"Mais là on va en sortir, avec l'avènement des plateformes et des nouveaux diffuseurs. Moi je crois beaucoup à la diffusion sur le Net, je crois beaucoup au réseau international, à la diffusion à l'international, je crois beaucoup à des plateformes très éditorialisées, radicalement axées sur des œuvres de création, qu'il y a une place pour des images intelligentes, et créatives au sens artistique, et c'est ça qu'il faut défendre. C'est un enjeu politique, intellectuel majeur, et il faut y aller à fond, quoi. » (Auteur-réalisateur, producteur, fondateur de Tënk, programmateur et cofondateur des États généraux du film documentaire)

Comme le résume explicitement le verb`tim ci-dessus, la plateforme de vidéo à la demande par abonnement Tënk revêt donc des enjeux tout à la fois symboliques, politiques, économiques et industriels. Si c'est la mobilisation d'un monde professionnel pour sa " survie » qui apparaît dans ce projet, la dimension industrielle est concrètement investie par ses fondateurs. Sa vocation est bien de s'insérer dans le jeu concurrentiel et de prendre part au processus industriel de fabrication des films documentaires. Cette dimension industrielle est ainsi alliée à la dimension symbolique, et non pas « mise à distance » d'elle (Noël, 2012, p. 79).

S'intéresser à une jeune plateforme de niche plutôt qu'aux modèles dominants tels que Netflix ou Amazon permet de mettre en évidence des enjeux spécifiques qui ne sont pas nés avec le numérique. Bien au contraire, ils réapparaissent avec

\footnotetext{
${ }^{6}$ En 2015-2016, lors de la mobilisation des professionnels du documentaire, un des enjeux débattus était de choisir ou non de s"llier à la fiction indépendante. L'appréhension de cette question est très variable selon les professionnels interrogés et leur carrière.
} 
Une plateforme au service d'un monde professionnel mobilisé : enjeux symboliques et économiques

lui et doivent être pris en compte lorsqu'on cherche à comprendre et à expliquer les rapports entre plateformes numériques et industries culturelles ainsi que les jeux concurrentiels qui en résultent.

La problématique quant à la viabilité de la plateforme semble aujourd'hui liée à deux dimensions. Du côté de la diffusion, c'est la question des usages, et la mutualisation des plateformes de niche semble être une piste de réponse. Du côté de la production, la législation du CNC quant aux modalités de financement des œuvres par les plateformes porte en germe l'hypothèse d'une nouvelle forme de marginalisation. Le financement apporté aux films par Tënk a été pensé sous la forme d'apports en industrie. Le CNC réserve cependant aujourd'hui l'accès au soutien automatique (donc économiquement structurant) à des œuvres dont une plateforme apporte $25 \%$ du budget du film... en numéraire. Cette législation, pensée pour contrer l'emprise des plateformes dominantes, contraint les acteurs de Tënk à adapter leur modèle économique, mais aussi à négocier à nouveau avec les institutions pour défendre le monde professionnel, son fonctionnement et ses frontières.

\section{Références}

Adorno, T. W. (1971). Erziehung zur Mündigkeit: Vorträge und Gespräche mit Hellmut 1959 bis 1969). Francfort, Allemagne : Suhrkamp.

Adorno T. W. et Horkheimer, M. (1947). L' di'lectique de I' $r$ ison. Paris, France : Gallimard.

Anderson, C. (2007). L' longue tr' îne. $L^{\prime}$ nouvelle économie est là. Paris, France : Pearson - Village mondial.

Barreau-Brouste, S. (2011). Arte et le document ire. De nouve 'ux enjeux pour l' cré tion. Lormont, France : Éditions Le bord de l'eau.

Barreau-Brouste, S. (2013). Le documentaire télévisé : enjeux d'une définition controversée. Le document'ire, un genre multiforme, e-dossier, Ina.

Becker, H. (1982) Les mondes de l"rt. Paris, France: Flammarion. 


\section{Marie-Eva LESAUNIER}

Becker, H. (1985). Outsiders. Études de sociologie de I' dévi nce. Paris, France : Métailié.

Benghozi, P.-J. (2006). Mutations et articulations contemporaines des industries culturelles. Dans X. Greffe (dir.), Cré'tion et diversité ' $u$ miroir des industries culturelles: 'ctes des Journées d'économie de I' culture des 12 et $13 j$ nvier 2016 (p. 129-152). Paris, France : ministère de la Culture, DEPS.

Benghozi, P.-J. et Benhamou, F. (2008). Longue traîne : levier numérique de la diversité culturelle?. Culture prospective, (1), 1-11.

Benghozi, P.-J. et Paris, T. (2003). De l'intermédiation à la prescription: le cas de la télévision. Revue $\mathrm{fr}^{\prime} n c^{\prime}$ ise de gestion, (142), 205-227.

Benjamin, W. (1936). L'œuvre d' rt à l'ère de s' reproductibilité technique. CEuvre I. Paris, France : Gallimard.

Blondiaux, M. et Barneaud, D. (2015). Le document ire sur internet, ses 'uteurs et ses publics. Étude Scam/Red Corner.

Bourdieu, P. (1992). Les règles de l"rt. Genèse et structure du ch `mp littér`ire. Paris, France : Seuil.

Bourreau, M., Maillard, S. et Moreau, F. (2015). Une analyse économique du phénomène de la longue traîne dans les industries culturelles. Revue $\mathrm{fr}^{\prime} n \mathrm{nç}^{\prime}$ ise d'économie, $X X X(2)$, 179-216.

Bullich, V. et Guignard T. (2014). Les plates-formes de contenus numériques: une nouvelle intermédiation?. Dans L. Jeanpierre et O. Roueff (dir.), L' culture et ses intermédi ires. $D$ 'ns les 'rts, le numérique et les industries cré tives (p. 201-210). Paris, France : Éditions des Archives contemporaines.

CNC (2017a, décembre). Observ toire de I' VàD. Étude prospective.

CNC (2017b, juin). Présent tion CNC. Le soutien à l' production document ire. Sunny side of the doc.

CNC (2018a). Le marché du documentaire. Les synthèses du CNC, (4). 
Une plateforme au service d'un monde professionnel mobilisé : enjeux symboliques et économiques

CNC (2018b). Les aides du CNC au documentaire. Les synthèses du CNC, (5).

CNC, CSA (2018). $L$ ' vidéo à $l$ ' dem 'nde $p^{\prime} r$ 'bonnement en Fr' nce : M'rché et str' tégies d" cteurs. Etude prospective.

Kilborne, Y. (2011). Le petit écran, média indépassable ? Du statut de la télévision chez les cinéastes documentaristes. Les enjeux de l'inform 'tion et de I' communic'tion, 12(1), 95-106.

Mairesse, F. et Rochelandet, F. (2015). Économie des 'rts et de I' culture. Paris, France : Armand Colin.

Noël, S. (2012). Maintenir l'économie à distance dans l'univers des biens symboliques: le cas de l'édition indépendante "critique ». Revue fr' $n c ̧$ ' ise de socio-économie, 2(10), 7392.

Reynaud-Cressent, B. (1982). La dynamique d'un oligopole avec frange: le cas de la branche d'édition de livres en France. Revue d'économie industrielle, (22), 61-71.

Ripoll, F. (2005). S'approprier l'espace... ou contester son appropriation? Une vue des mouvements sociaux contemporains. Norois, (195), 29-42.

Sauguet, É. (2007). La diffusion des films documentaires: la construction des frontières d'une activité artistique (enquête). Terr' ins \& tr' $v^{`} u x, 13(2), 31-50$.

Shibutani, T. (1955). Reference Groups as Perspectives. Americ' $n$ Journ' I of Sociology, (60), 562-569.

Strauss, A. (1992). L’ tr’ me de I’ négoci tion. Paris, France : L'Harmattan.

Voirol, O. (2001). Retour sur l'industrie culturelle. Rése`ux, (166), 125-157. 